\title{
Effect of BRCA1 on the Concurrent Chemoradiotherapy Resistance of Cervical Squamous Cell Carcinoma Based on Transcriptome Sequencing Analysis
}

\author{
Xin Wen ${ }^{D}$, ${ }^{1}$ Shui Liu $\mathbb{D}^{2},{ }^{2}$ and Manhua Cui $\mathbb{D}^{1}$ \\ ${ }^{1}$ Department of Gynecology and Obstetrics, The Second Hospital of Jilin University, Changchun, Jilin, China \\ ${ }^{2}$ The Second Hospital of Jilin University, Changchun, Jilin, China \\ Correspondence should be addressed to Manhua Cui; cuimanhua@126.com
}

Received 22 December 2019; Revised 29 May 2020; Accepted 3 June 2020; Published 24 June 2020

Academic Editor: Nobuo Kanazawa

Copyright (๑) 2020 Xin Wen et al. This is an open access article distributed under the Creative Commons Attribution License, which permits unrestricted use, distribution, and reproduction in any medium, provided the original work is properly cited.

\begin{abstract}
Background. Cervical squamous cell carcinoma (CSCC) is the main pathological type of cervical cancer, accounting for $80 \%-85 \%$ of cervical cancer. Owing to concurrent chemoradiotherapy (CCRT) resistance in a subset of CSCC patients, the treatment response is often unsatisfactory. Identifying predictors and therapeutic targets related to cisplatin-based CCRT resistance in CSCC is critical. Methods. We reanalyzed GSE56363, an mRNA dataset from the GEO database with 21 patients with locally advanced CSCC, to identify differentially expressed genes (DEGs) related to CCRT resistance. The hub genes were screened from the proteinprotein interaction network of DEGs using cytoHubba plug-in of Cytoscape software. Transcriptome sequencing technology was used to compare differential expression between $\mathrm{SiHa}$ cells overexpressing BRCA1 compared with control SiHa cells. Functional annotation for DEGs and gene set enrichment analysis (GSEA) was performed to identify DEG-enriched relative signaling pathways to examine the molecular mechanisms of BRCA1 in CCRT resistance of CSCC. qPCR was used to verify the expression of key genes in SiHa/DDP cells. Results. A total of 609 DEGs including 223 upregulated DEGs and 386 downregulated DEGs were identified between the complete response to CCRT (CR) and noncomplete response to CCRT (NCR) CSCC patients based on the GSE56363 dataset. Ten hub genes with the highest degrees were identified via the plug-in CytoHubba in Cytoscape: BRCA1, CDCA8, ASPM, CDC45, RAD51, HMMR, CENPF, EXO1, DTL, and ZWINT genes, and BRCA1 ranked first. Through transcriptome sequencing analysis based on GSE141558, 1344 DEGs were identified in BRCA1overexpressing SiHa cells, including 824 upregulated DEGs and 520 downregulated DEGs. GSEA results showed that CCRTresistance related signaling pathways, such as the JAK/STAT signaling pathway and the WNT signaling pathway, were differentially enriched in BRCA1-expressing SiHa cells. STAT1, STAT2, and CCND1 were screened as the differentially expressed target genes of BRCA1 and may correlate with resistance of CSCC. qPCR results showed that only STAT1 was significantly increased in SiHa cells with GV230-BRCA1 plasmid transfection. Conclusion. BRCA1 overexpression in SiHa cells may upregulate STAT1 to activate the JAK/STAT signaling pathway, suggesting a mechanism for enhanced CCRT resistance.
\end{abstract}

\section{Introduction}

Cervical cancer (CC) ranks fourth for both incidence and mortality among cancers in women worldwide, and there were an estimated 570,000 new cases and 311,000 deaths related to CC in 2018 [1]. Cervical squamous cell carcinoma (CSCC) is the main pathological type of CC, accounting for $80 \%-85 \%$ of all CC cases. Approximately two-thirds of CSCC patients are diagnosed at medium or advanced stage, and the standard treatment is a concurrent chemoradiother- apy (CCRT) regimen consisting of cisplatin-based chemotherapy, external beam radiotherapy, and brachytherapy [2]. However, owing to CCRT resistance in a subset of patients, the treatment response is often unsatisfactory. Therefore, identifying efficient predictors and therapeutic targets related to CCRT is critical to improve the prognosis of CSCC patients.

With the rise of bioinformatics and high-throughput sequencing technologies, cancer development mechanisms, drug resistance, and prognosis can be better explained at 
TABLE 1: Primer sequences used for qRT-PCR amplification.

\begin{tabular}{lc}
\hline Primer & Sequences \\
\hline BRCA1 & $5^{\prime}$-GACAGTCAGCCGCATCTTCT-3' \\
& $5^{\prime}$-ACCAAATCCGTTGACTCCGA-3' \\
STAT1 & $5^{\prime}$-ATCAGGCTCAGTCGGGGAATA-3' \\
& $5^{\prime}$-TGGTCTCGTGTTCTCTGTTCT-3' \\
STAT2 & $5^{\prime}$-CCAGCTTTACTCGCACAGC-3' \\
& $5^{\prime}$-AGCCTTGGAATCATCACTCCC-3' \\
CCND1 & $5^{\prime}$-GCTGCGAAGTGGAAACCATC-3' \\
& $5^{\prime}$-CCTCCTTCTGCACACATTTGAA-3' \\
GAPDH & $5^{\prime}$-GACAGTCAGCCGCATCTTCT-3' \\
& $5^{\prime}$-ACCAAATCCGTTGACTCCGA-3' \\
\hline
\end{tabular}

the genetic level $[3,4]$. These findings will contribute to understanding individual differences and tumor heterogeneity in cancer patients. These technologies have been applied to investigate predictors and therapeutic targets of radiotherapy and chemotherapy resistance in many tumor types [5-7]. However, relatively few studies have examined CCRT resistance in CSCC $[8,9]$.

The BRCA1 tumor suppressor responds to DNA damage by participating in DNA repair, mRNA transcription, cell cycle regulation, protein ubiquitination, and other cellular pathways $[10,11]$. The DNA repair ability of damaged cells improves with increased intracellular BRCA1 expression, resulting in drug resistance. Multiple studies have shown that the expression level of BRCA1 gene in cancers is negatively correlated with the sensitivity to platinum drugs [12-14] and sensitivity to radiotherapy [15-17].

In the current study, we screened the differentially expressed genes (DEGs) related to CCRT resistance of CSCC and found that BRCA1 overexpression enhanced CCRT resistance in CSCC. Subsequently, we performed transcriptome sequencing to explore the potential mechanisms by which BRCA1 may enhance resistance in CSCC. Our findings may provide theoretical support and new strategies for the diagnosis and treatment of CSCC patients with CCRT resistance.

\section{Materials and Methods}

2.1. Identification of DEGs Related to CCRT Resistance in CSCC Based on the GEO Dataset. The GSE56363 database of CSCC patients was downloaded from the NCBI-GEO database [18]. The CSCC patients were treated by CCRT, and cisplatin-based treatments were used as chemotherapeutics. Patients were divided into two groups: complete response to CCRT (CR) and noncomplete response to CCRT (NCR). The DEGs between NCR and CR patients were reanalyzed using limma package in $\mathrm{R}$ [19]. False discovery rate (FDR) $<0.05$ and $\mid$ LogFoldChange(FC) $\mid>1$ were considered as the cut-off criterion.
2.2. Gene Ontology (GO) Enrichment Analysis and Construction of the Protein-Protein Interaction Network. We performed GO enrichment for the DEGs related to CCRT resistance in CSCC by clusterProfiler package [20]. The STRING database (https://string-db.org/) is an online resource that provides known direct and indirect proteinprotein interaction (PPI). We mapped the DEGs to STRING [21] to reveal the PPI information and visualized the PPI network using Cytoscape 3.7.2 [22]. The combined scores ( interaction scores) $>0.4$ were considered as significant. We then analyzed the PPI network to screen the hub genes using the cytoHubba plug-in of Cytoscape software [23].

We selected the top 10 DEGs with the highest degrees for further analysis. The receiver operating characteristic (ROC) curves of the hub genes were generated by the pROC package in $\mathrm{R}$ [24]. The violin plots of the hub gene expression were developed by the ggplot2 package in R [25]. Heat maps of the hub gene expression were developed by the pheatmap package in $\mathrm{R}[26]$.

2.3. Cells, Cell Culture, and Plasmid Transfection. The SiHa human CSCC cell line was purchased from Cell Bank of the Chinese Academy of Sciences, and SiHa/DDP cells were purchased from Fenghui Bio-Technology Limited. The cells were cultured in DMEM medium (Gibco, USA) supplemented with $10 \%$ fetal bovine serum (Sangon, China) and $1 \%$ antibiotic-antimitotic solution (Gibco). The GV230BRCA1 and GV230 plasmids were purchased from GeneChem (Shanghai, China). SiHa cells were transfected using Lipofectamine 3000 reagent (Invitrogen, USA) following the manufacturer's protocol. Transfected cells were incubated for $48 \mathrm{~h}$ before further analysis.

2.4. Reverse Transcription and Quantitative Real-Time PCR. Total RNA was extracted using TRIzol (Sangon, Shanghai, China) according to the manufacturer's protocols. The quantity and quality of RNA were measured using the NanoDrop 2000 spectrophotometer (Thermo Scientific, USA). qRTPCR was performed using EasyScript ${ }^{\circledR}$ First-Strand cDNA Synthesis SuperMix (TransGen Biotech, Beijing, China) and 2x SG Fast qPCR Master Mix (Sangon Biotech, China) with the Roche LightCycler480 PCR system. The $2^{-\Delta \Delta C T}$ method was used to calculate relative expression. The primer sequences are listed in Table 1. Experiments were performed in triplicate.

2.5. Transcriptome Sequencing of $m R N A s$ and Differential Expression Analysis. Sequencing libraries were produced using the Hieff NGS ${ }^{\mathrm{TM}}$ MaxUp Dual-mode mRNA Library Prep Kit for Illumina (YEASEN, Shanghai, China) based on the instructions from the manufacturer. First, total RNA was quantified using the Qubit 2.0 RNA Assay Kit (Life, USA) to determine the amount of total RNA added to the library construction. Purification and fragmentation of mRNA were performed using the mRNA Capture Beads and Frag/Prime buffer. Synthesis and purification of double-stranded DNA were conducted using 1st Strand Synthesis Reaction Buffer, 2nd Strand Synthesis Buffer, and Hieff $\mathrm{NGS}^{\mathrm{TM}}$ DNA Selection Beads before PCR. Amplification and 


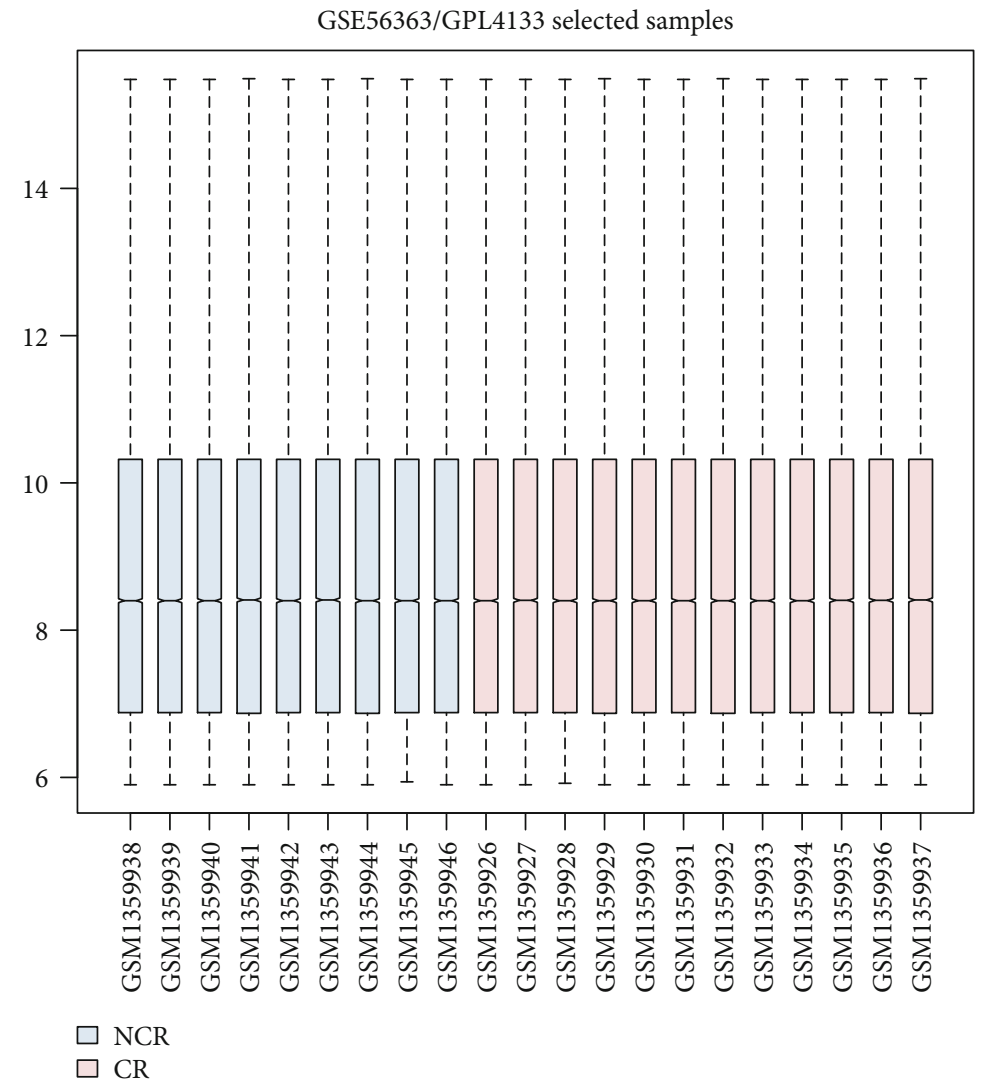

(a)

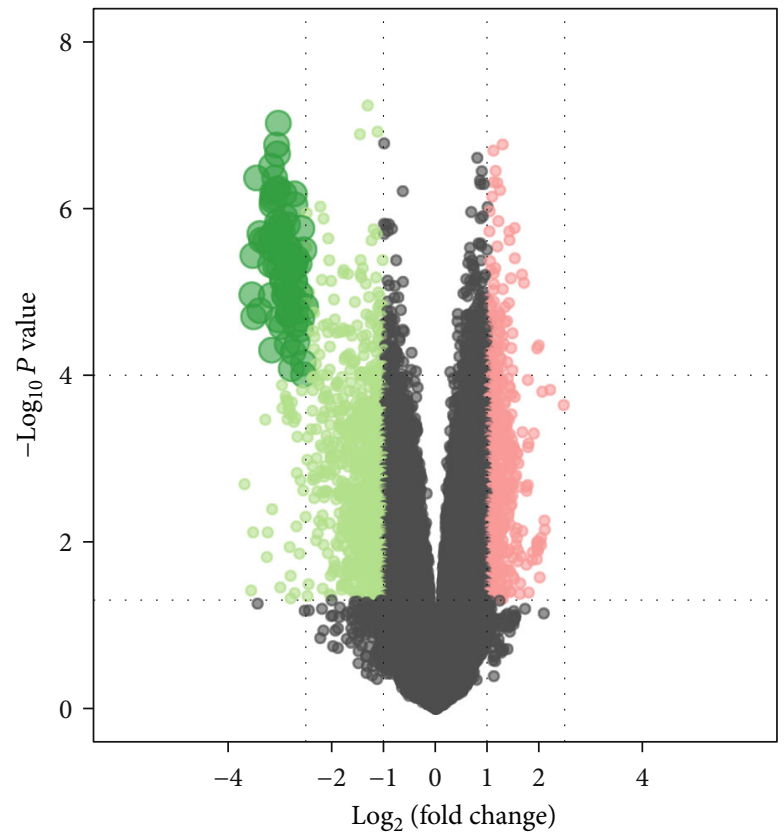

(b)

FIGURE 1: Processing of microarray data and identification of DEGs. (a) Boxplot of normalized expression values for GSE56363. The dotted line in the middle of each box represents the median of each sample, and its distribution among samples indicates the level of normalization of the data, with a straight line revealing a fair normalization level. (b) Volcano plot of DEGs based on GSE56363. The upregulated DEGs in NCR CSCC patients were signed in red, and downregulated DEGs were signed in green. 
TABLE 2: The significantly enriched analysis of DEGs in NCR CSCC patients.

\begin{tabular}{|c|c|c|c|c|}
\hline Category & Term & Description & Adjusted $P$ value & Count \\
\hline BP & GO:0009615 & Response to virus & $2.19 E-17$ & 67 \\
\hline BP & GO:0060337 & Type I interferon signaling pathway & $2.19 E-17$ & 35 \\
\hline $\mathrm{BP}$ & GO:0071357 & Cellular response to type I interferon & $2.19 E-17$ & 35 \\
\hline BP & GO:0034340 & Response to type I interferon & $1.36 E-16$ & 35 \\
\hline BP & GO:0034341 & Response to interferon-gamma & $4.28 E-16$ & 50 \\
\hline BP & GO:0051607 & Defense response to virus & $1.64 E-15$ & 53 \\
\hline BP & GO:1903900 & Regulation of viral life cycle & $1.02 E-14$ & 40 \\
\hline BP & GO:0071346 & Cellular response to interferon-gamma & $8.74 E-14$ & 44 \\
\hline BP & GO:0060333 & Interferon-gamma-mediated signaling pathway & $1.01 E-13$ & 31 \\
\hline $\mathrm{BP}$ & GO:0050792 & Regulation of viral process & $1.64 E-12$ & 42 \\
\hline CC & GO:0071556 & Integral component of lumenal side of endoplasmic reticulum membrane & $7.23 E-06$ & 12 \\
\hline CC & GO:0098553 & Lumenal side of endoplasmic reticulum membrane & $7.23 E-06$ & 12 \\
\hline CC & GO:0005925 & Focal adhesion & $6.34 E-05$ & 49 \\
\hline $\mathrm{CC}$ & GO:0005924 & Cell-substrate adherens junction & $6.34 E-05$ & 49 \\
\hline CC & GO:0005788 & Endoplasmic reticulum lumen & $6.34 E-05$ & 40 \\
\hline $\mathrm{CC}$ & GO:0030055 & Cell-substrate junction & $6.90 E-05$ & 49 \\
\hline CC & GO:0012507 & ER to Golgi transport vesicle membrane & $1.29 E-04$ & 15 \\
\hline CC & GO:0030176 & Integral component of endoplasmic reticulum membrane & $2.83 E-04$ & 24 \\
\hline CC & GO:0042611 & MHC protein complex & $3.75 E-04$ & 9 \\
\hline $\mathrm{CC}$ & GO:0030662 & Coated vesicle membrane & $3.75 E-04$ & 26 \\
\hline MF & GO:0005201 & Extracellular matrix structural constituent & $8.84 E-08$ & 22 \\
\hline MF & GO:0019838 & Growth factor binding & $9.67 E-07$ & 19 \\
\hline MF & GO:0005539 & Glycosaminoglycan binding & $9.67 E-07$ & 24 \\
\hline MF & GO:0005178 & Integrin binding & $9.67 E-07$ & 18 \\
\hline MF & GO:0008201 & Heparin binding & $4.38 E-05$ & 18 \\
\hline MF & GO:0004714 & Transmembrane receptor protein tyrosine kinase activity & $6.88 E-04$ & 10 \\
\hline MF & GO:1901681 & Sulfur compound binding & $6.88 E-04$ & 20 \\
\hline MF & GO:0050839 & Cell adhesion molecule binding & $1.36 E-03$ & 30 \\
\hline MF & GO:0030020 & Extracellular matrix structural constituent conferring tensile strength & $3.32 E-03$ & 7 \\
\hline MF & GO:0019199 & Transmembrane receptor protein kinase activity & $3.55 E-03$ & 10 \\
\hline
\end{tabular}

purification of the library were performed by PCR method with the 2x Super Canace ${ }^{\mathrm{TM}}$ High-Fidelity Mix, primer mix, and Hieff NGS ${ }^{\mathrm{TM}}$ DNA Selection Beads. PCR products were collected and assessed using the Qubit2.0 DNA Test Kit (Life). Transcriptome sequencing was performed by Sangon Biotech Co. Ltd. (Shanghai, China). DEGs were detected by the negative binomial distribution test using the DESeq $\mathrm{R}$ package [27]. FDR $<0.05$ and $|\operatorname{LogFC}|>1$ were the cut-off criterion. Each group sample for transcriptome sequencing contained three biological replicates.

2.6. euKaryotic Ortholog Groups (KOG) Functional Annotation and Gene Set Enrichment Analysis (GSEA). KOG is the NCBI annotation system based on the direct homologous relationship of genes, in which Clusters of Orthologous Groups of proteins (COG) are targeted at pro- karyotes for eukaryotes. KOG currently has 4852 classifications and can be combined with evolutionary relationships to classify homologous genes from different species into different ortholog clusters.

To explore potential mechanisms underlying the effect of BRCA1 in CCRT resistance in CSCC, GSEA was performed to detect whether a priori defined set of genes showed statistically significant differential expression between the BRCA1 overexpression group and negative control group using Broad Institute software [28]. Gene sets with a nominal $P$ value $<0.05$ and FDR $<0.25$ were considered significantly enriched.

Transcriptional Regulatory Relationships Unraveled by Sentenced-based Text mining Version 2.0 database (TRRUST) (https://www.grnpedia.org/trrust/) is a database based on the literature to reflect the relationship between transcriptional 


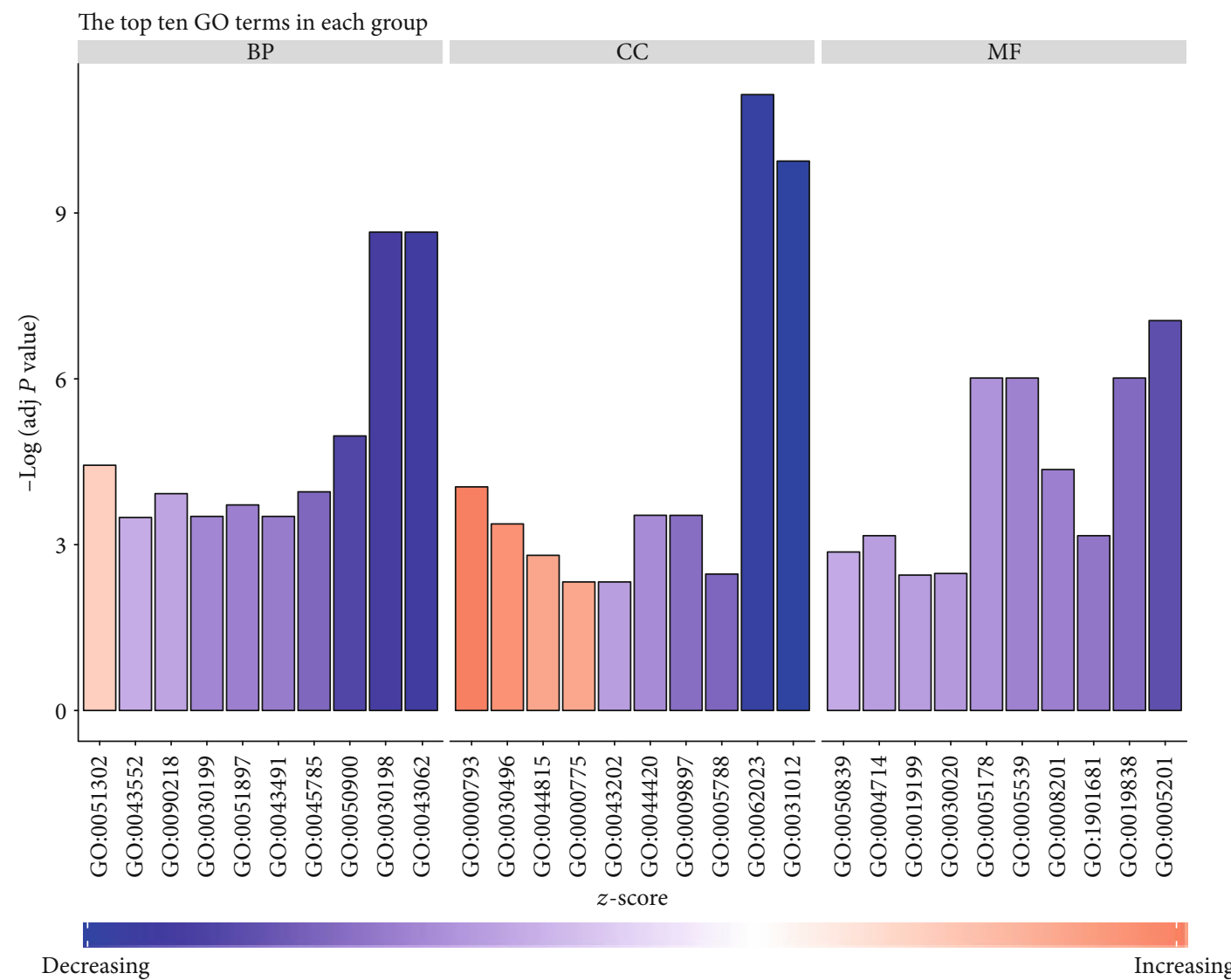

Figure 2: GO enrichment analysis of DEGs. The top 10 GO terms in each group were ranked by adjusted $P$ value. The gradual color represents the $z$-score.

regulations [29]. To screen the mechanisms of BRCA1 in resistance in CSCC, we searched the targets of BRCA1 using the TRRUST Version 2 database.

2.7. Statistical Analysis. Statistical analysis was performed using GraphPad Prism 7.0 (GraphPad Software Inc., USA). FDR controlling was used to correct $P$ values with the Benjamini Hochberg algorithm implemented in $\mathrm{R}$ 3.5.1 suite (Lucent Technologies). FDR $<0.05$ and $P$ value $<0.05$ were considered statistically significant.

\section{Results}

3.1. Processing of Microarray Data and Identification of DEGs from the GEO Dataset. The GSE56363 dataset was normalized and the results are shown in Figure 1(a). A total of 21 CSCC patients at advanced stage including 9 NCR and 12 $\mathrm{CR}$ patients were analyzed. We obtained $609 \mathrm{DEGs}$ including 223 upregulated DEGs and 386 downregulated DEGs in NCR patients compared with CR patients (Figure 1(b)).

3.2. GO Enrichment Analysis for DEGs. To obtain the function of the DEGs related to CCRT in CSCC, enrichment analysis of DEGs was performed using clusterProfiler in R. As shown in Table 2 and Figure 2, in the biological process group, the DEGs were most enriched in Response to virus. In the cellular component group, the DEGs were most enriched in the Integral component of lumenal side of endoplasmic reticulum membrane. In the molecular function group, the DEGs were most enriched in extracellular matrix structural constituent.

3.3. Construction of the PPI Network and Screening and Analysis of Hub Genes. The PPI of the DEGs was collected by STRING and visualized in Cytoscape software. We evaluated the degree and betweenness centrality in the PPI network through the CytoHubba plug-in and identified the top 10 hub genes with the highest degrees: BRCA1, CDCA8, ASPM, CDC45, RAD51, HMMR, CENPF, EXO1, DTL, and ZWINT (Figure 3). Among them, BRCA1 has the highest degrees. The ROC curves of the hub genes all indicated favorable diagnostic value in CSCC patients with CCRT resistance (Figure 4).

3.4. Validation of BRCA1 Expression. The $\mathrm{qPCR}$ results showed that the expression level of BRCA1 mRNA was significantly upregulated in SiHa/DDP cells compared with SiHa cells (Figure 5(a)). Furthermore, the expression level of BRCA1 was significantly increased in $\mathrm{SiHa}$ cell lines transfected with the GV230-BRCA1 plasmid (OV-BRCA1 group) compared with cells transfected with empty vector (NC group) (Figure 5(b)). 


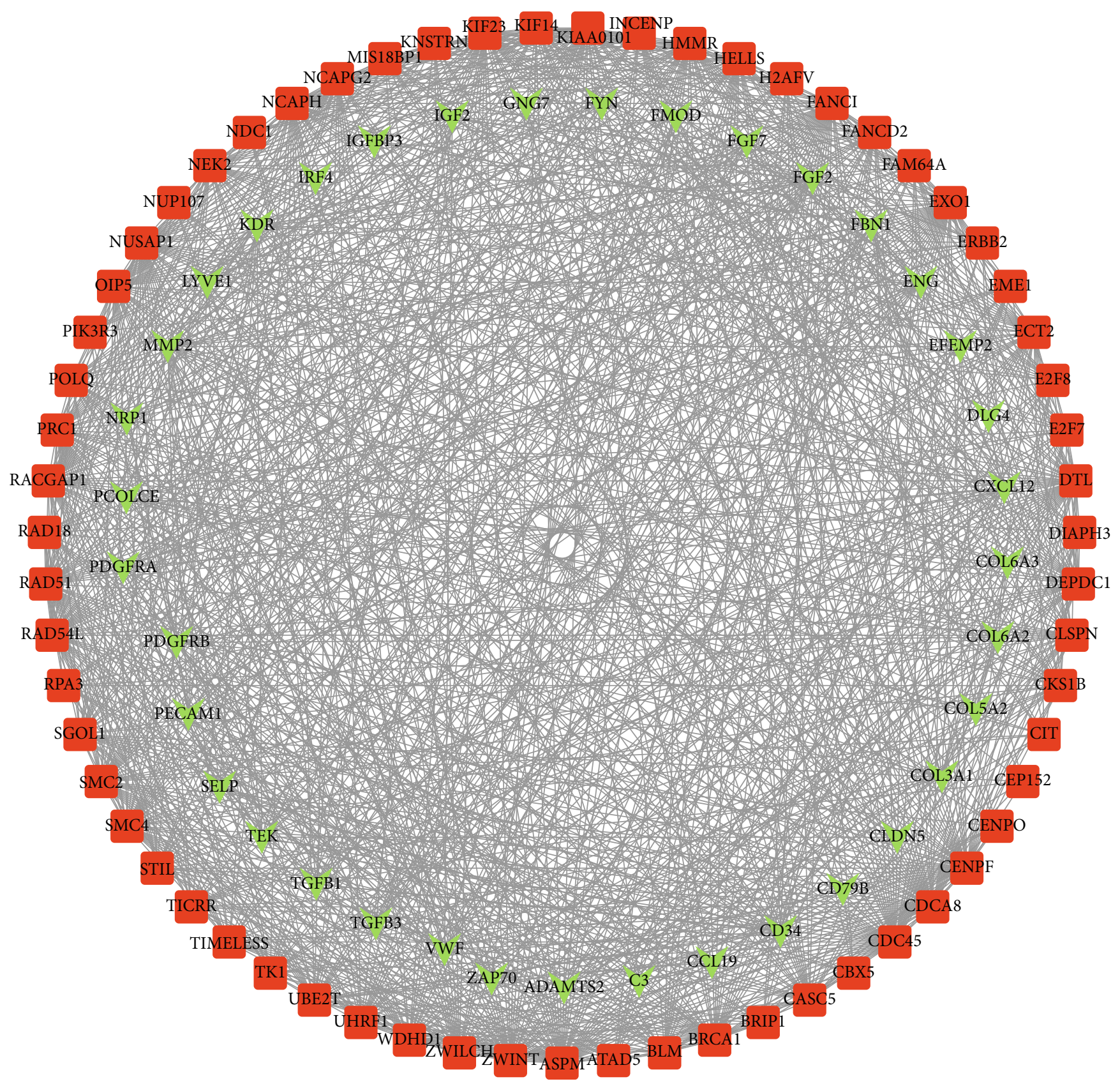

(a)

Figure 3: Continued. 


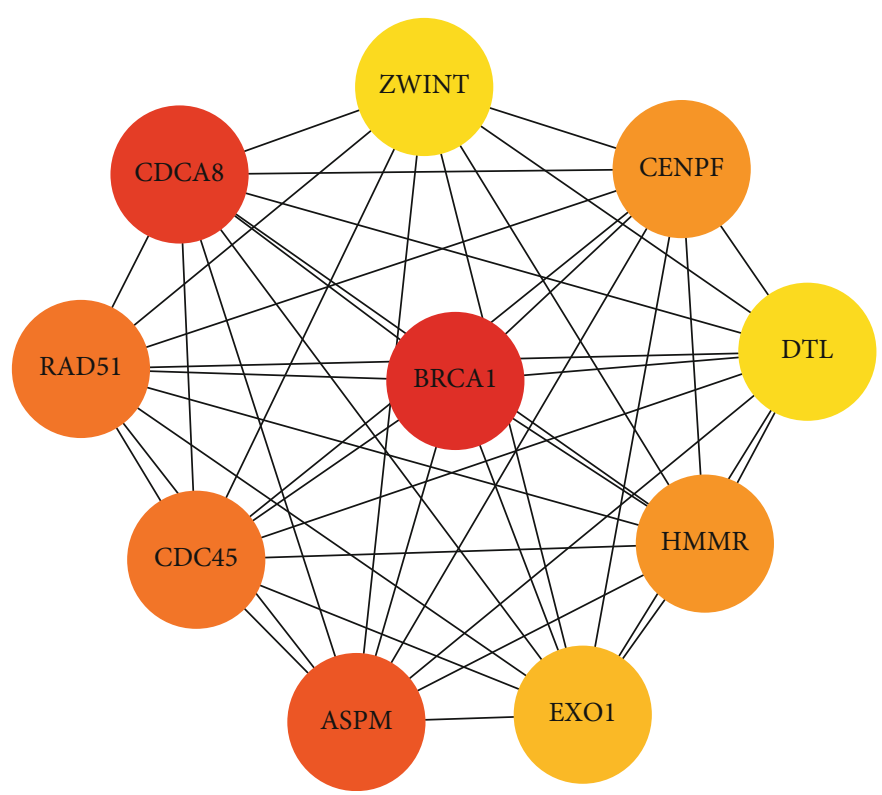

(b)

FIGURE 3: PPI networks. (a) PPI network of top 100 DEGs with the highest degrees. Red nodes denote upregulated genes, while green nodes denote downregulated genes. (b) PPI network of the hub genes. The gradual color represents the degree value.

3.5. Identification and GO and KOG Enrichment of DEGs in SiHa Cells with BRCA1 Overexpression Plasmid Transfection. After removing reads with adapters, unknown nucleotides, and low-quality reads of sequencing, we acquired a total of $15.79 \mathrm{~Gb}$ clean data. The Q30 percentage of all samples was more than $90 \%$ (Table S1). The transcriptome sequencing data have been uploaded to the GEO database, and the accession number is GSE141558. We normalized the read count data using the FPKM method. Using FDR $<0.05$ and $|\log \mathrm{FC}|>1$ as the screening criterion in the process of gene identification, the transcriptome sequencing analysis identified 1344 DEGs between the OV-BRCA1 and NC groups, including 824 upregulated DEGs and 520 downregulated DEGs (Table S2).

The enrichment analysis results showed that in the biological process group, the DEGs were most enriched in response to virus; in the cellular component group, the DEGs were most enriched in the nucleosome; and in the molecular function group, the DEGs were most enriched in transferase activity transferring pentosyl groups Table 3 and Figure 6).

We also performed KOG annotation to reveal the function distribution of the DEGs to clarify the embodiment of sample differences in gene function. The results of the DEGs classified on the KOG system are shown in Figure 7.

3.6. GSEA Identifies a Cisplatin Resistance-Related Signaling Pathway. GSEA results revealed a positive correlation of the DEGs in the OV-BRCA1 group with CCRT resistancerelated signaling pathways, such as the WNT signaling pathway and the JAK/STAT signaling pathway (Figures 8(a), $8(\mathrm{~b}), 8(\mathrm{~d})$, and $8(\mathrm{e}))$. We intersected the key genes in the WNT and JAK/STAT signaling pathway based on the GSEA results and the DEGs from the BRCA1 overexpression group and obtained 5 key DEGs in the WNT signaling pathway and
14 key DEGs in the JAK/STAT signaling pathway. The correlation analysis indicated a strong positive correlation between BRCA1 and the key DEGs in the WNT and JAK/STAT signaling pathways (Figures 8(c) and 8(f)).

3.7. BRCA1 May Affect CCRT Resistance via the JAK/STAT Signaling Pathway. To more closely examine the mechanisms of BRCA1 in CCRT resistance in CSCC, we intersected the key genes in the WNT and JAK/STAT signaling pathway and target genes of BRCA1 based on the TRRUST database (Figure 9(a)). We found that BRCA1 may upregulate the expressions of STAT1, STAT2, and CCND1, three key genes in the JAK/STAT and WNT signaling pathway (Figure 9(b)). We performed qPCR verification, and the results showed that only STAT1 was significantly increased in SiHa cells with GV230-BRCA1 plasmid transfection (Figure 9(c)). These findings showed that overexpression of BRCA1 in CSCC cells may enhance resistance to CCRT through activating the JAK/STAT signaling pathway via upregulating STAT1.

\section{Discussion}

CCRT is the standard therapy for locally advanced CSCC; however, the development of intrinsic or acquired CCRT resistance dramatically decreases its clinical effectiveness. To investigate efficient biomarkers that could predict the response to CCRT and serve as potential therapeutic targets for CSCC patients, we performed comprehensive analysis of resistance in CSCC based on a GEO dataset and transcriptome sequencing. First, we reanalyzed the GSE56363 dataset and identified 609 DEGs including 223 upregulated DEGs and 386 downregulated DEGs between NCR and CR patients [18]. We performed GO enrichment for DEGs related to resistance in CSCC and identified 10 hub genes 


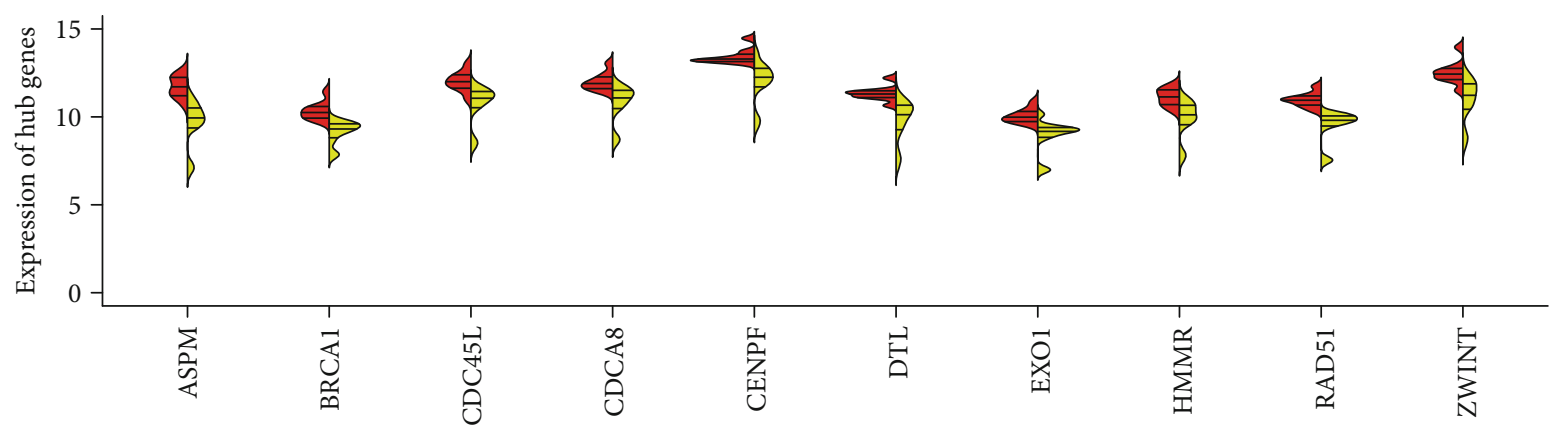

(a)

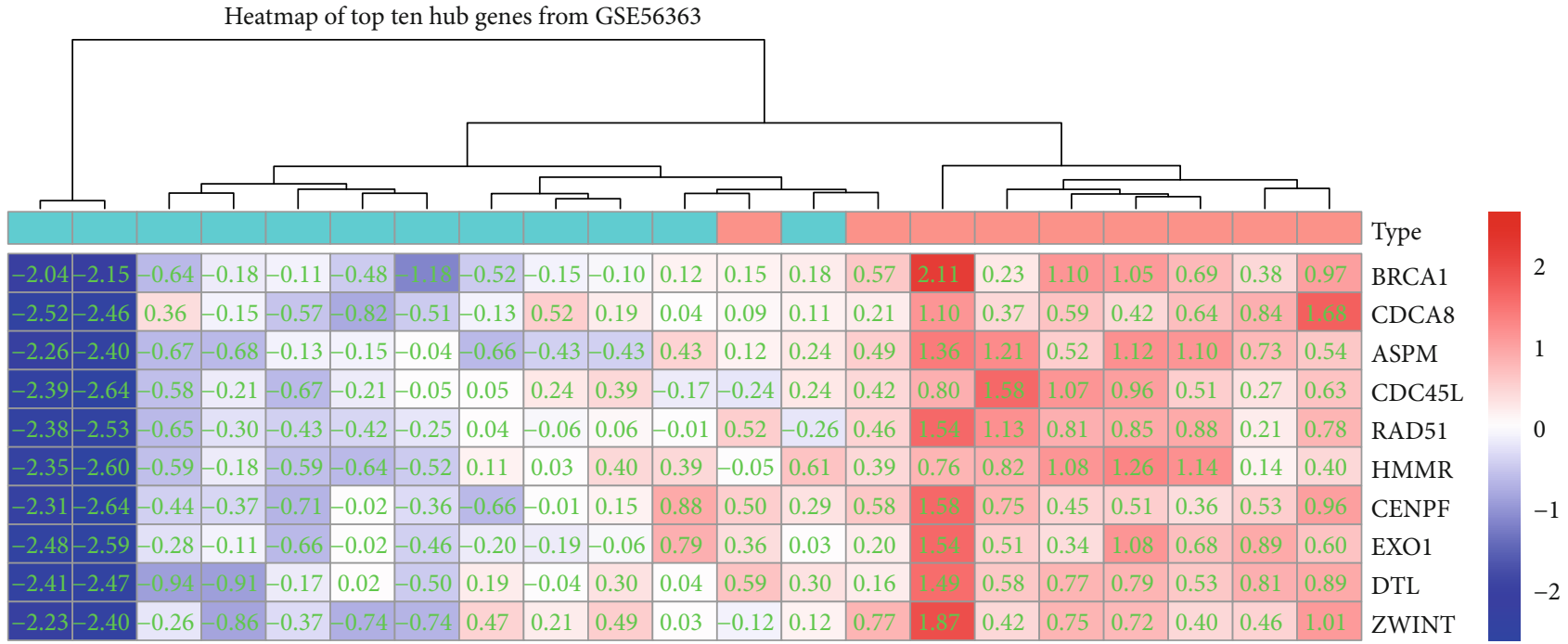

CR

NCR
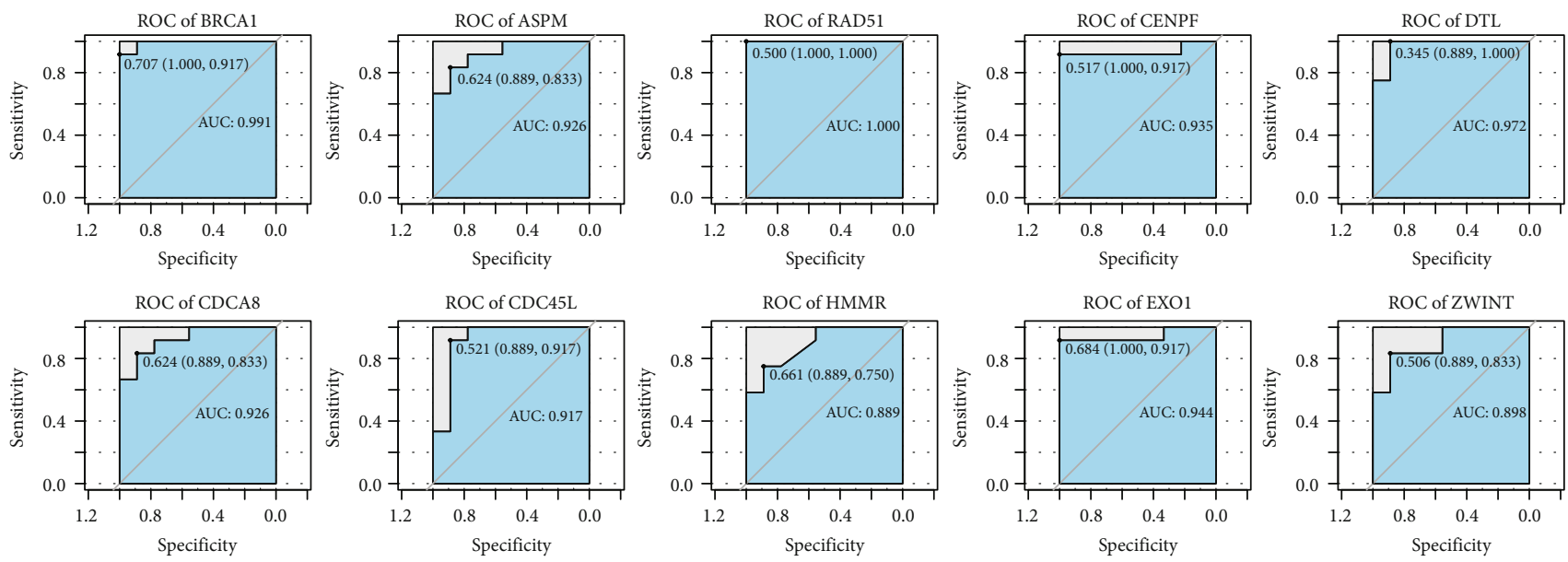

(c)

FIGURE 4: Comprehensive analysis of the hub genes. (a) Violin plots for different expressions of the hub genes between NCR and CR patients.

(b) Heat maps of the hub genes in CSCC. (c) ROC curves of the hub genes sorted by AUC. NCR: noncomplete response; CR: complete response; ROC: receiver operating characteristic; AUC: area under the curve. 


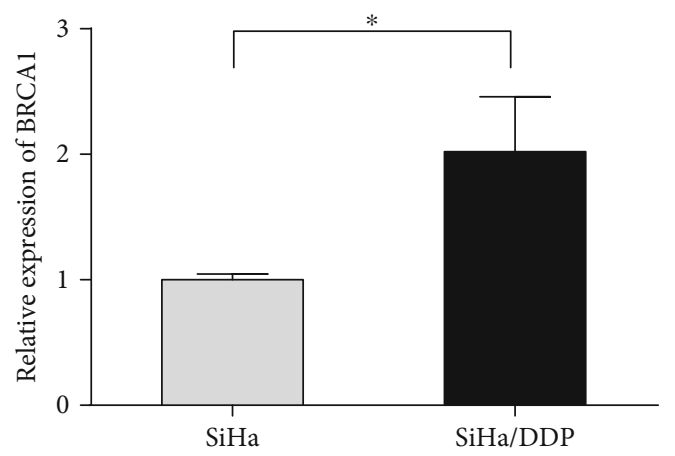

(a)

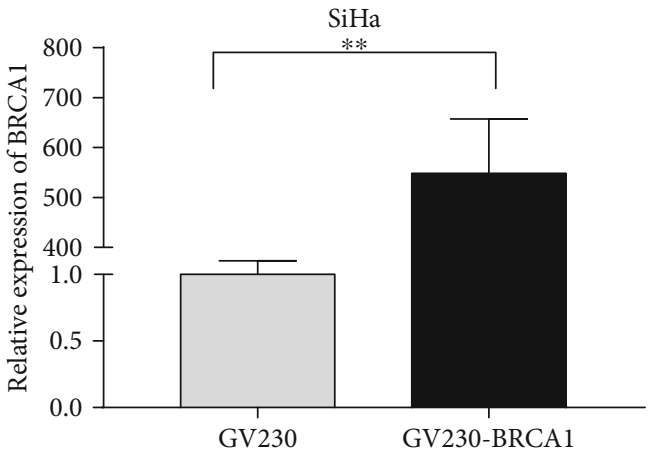

(b)

Figure 5: qRT-PCR. (a) The expression of BRCA1 by qRT-PCR between the SiHa/DDP group and the SiHa group. (b) The expression of BRCA1 in the SiHa cell with GV230-BRCA1 or GV230 transfection. $* P<0.05, * * P<0.01$.

TABLE 3: The significantly enriched analysis of DEGs between OV-BRCA1 and NC SiHa cells.

\begin{tabular}{|c|c|c|c|c|}
\hline Category & Term & Description & Adjusted $P$ value & Count \\
\hline $\mathrm{BP}$ & GO:0051607 & Defense response to virus & $4.34 E-25$ & 50 \\
\hline $\mathrm{BP}$ & GO:0009615 & Response to virus & $1.13 E-24$ & 57 \\
\hline $\mathrm{BP}$ & GO:0060337 & Type I interferon signaling pathway & $2.42 E-19$ & 29 \\
\hline $\mathrm{BP}$ & GO:0071357 & Cellular response to type I interferon & $2.42 E-19$ & 29 \\
\hline $\mathrm{BP}$ & GO:0034341 & Response to interferon-gamma & $7.82 E-19$ & 40 \\
\hline $\mathrm{BP}$ & GO:0071346 & Cellular response to interferon-gamma & $7.98 E-19$ & 38 \\
\hline $\mathrm{BP}$ & GO:0034340 & Response to type I interferon & $7.98 E-19$ & 29 \\
\hline $\mathrm{BP}$ & GO:0060333 & Interferon-gamma-mediated signaling pathway & $4.05 E-17$ & 27 \\
\hline $\mathrm{BP}$ & GO:1903900 & Regulation of viral life cycle & $1.13 E-15$ & 31 \\
\hline $\mathrm{BP}$ & GO:0048525 & Negative regulation of viral process & $8.16 E-15$ & 25 \\
\hline $\mathrm{CC}$ & GO:0000786 & Nucleosome & $2.38 E-11$ & 31 \\
\hline CC & GO:0044815 & DNA packaging complex & $1.05 E-10$ & 31 \\
\hline $\mathrm{CC}$ & GO:0032993 & Protein-DNA complex & $3.98 E-07$ & 36 \\
\hline CC & GO:0071556 & Integral component of lumenal side of endoplasmic reticulum membrane & $4.23 E-06$ & 12 \\
\hline $\mathrm{CC}$ & GO:0098553 & Lumenal side of endoplasmic reticulum membrane & $4.23 E-06$ & 12 \\
\hline $\mathrm{CC}$ & GO:0005788 & Endoplasmic reticulum lumen & $4.81 E-05$ & 41 \\
\hline $\mathrm{CC}$ & GO:0005925 & Focal adhesion & $8.43 E-05$ & 49 \\
\hline $\mathrm{CC}$ & GO:0000788 & Nuclear nucleosome & $8.43 E-05$ & 12 \\
\hline $\mathrm{CC}$ & GO:0005924 & Cell-substrate adherens junction & $9.24 E-05$ & 49 \\
\hline $\mathrm{CC}$ & GO:0030055 & Cell-substrate junction & $1.10 E-04$ & 49 \\
\hline MF & GO:0016763 & Transferase activity transferring pentosyl groups & $1.26 E-03$ & 13 \\
\hline MF & GO:0003950 & NAD+ ADP-ribosyltransferase activity & $1.26 E-03$ & 9 \\
\hline MF & GO:0002020 & Protease binding & $1.54 E-02$ & 20 \\
\hline MF & GO:0005125 & Cytokine activity & $1.54 E-02$ & 29 \\
\hline
\end{tabular}

with the highest degrees from the PPI network, including BRCA1, CDCA8, ASPM, CDC45, RAD51, HMMR, CENPF, EXO1, DTL, and ZWINT genes. We next performed transcriptome sequencing of SiHa cells transfected with GV230BRCA1 or GV230 plasmid and performed functional annotation for DEGs based on GO and KOG database. Finally, we performed GSEA to identify CCRT resistance related signaling pathways to investigate potential molecular mechanisms of resistance in CSCC. STAT1, STAT2, and CCND1 were selected as the differentially expressed target genes of BRCA1 in the WNT and JAK/STAT signaling pathway and may correlate with the CCRT resistance of CSCC. 


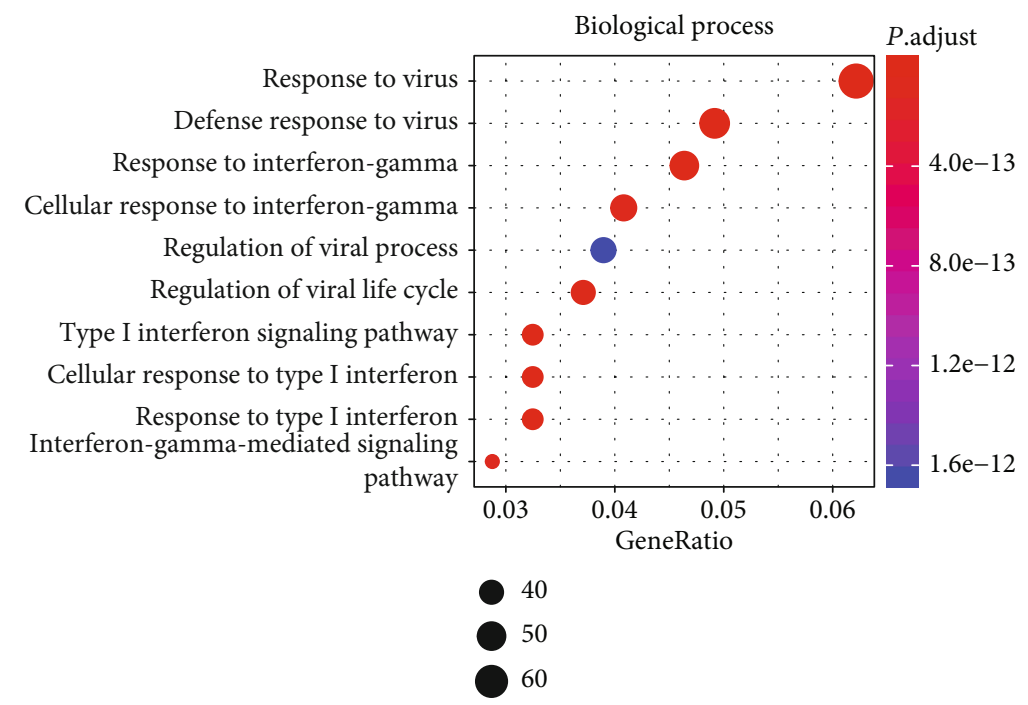

(a)

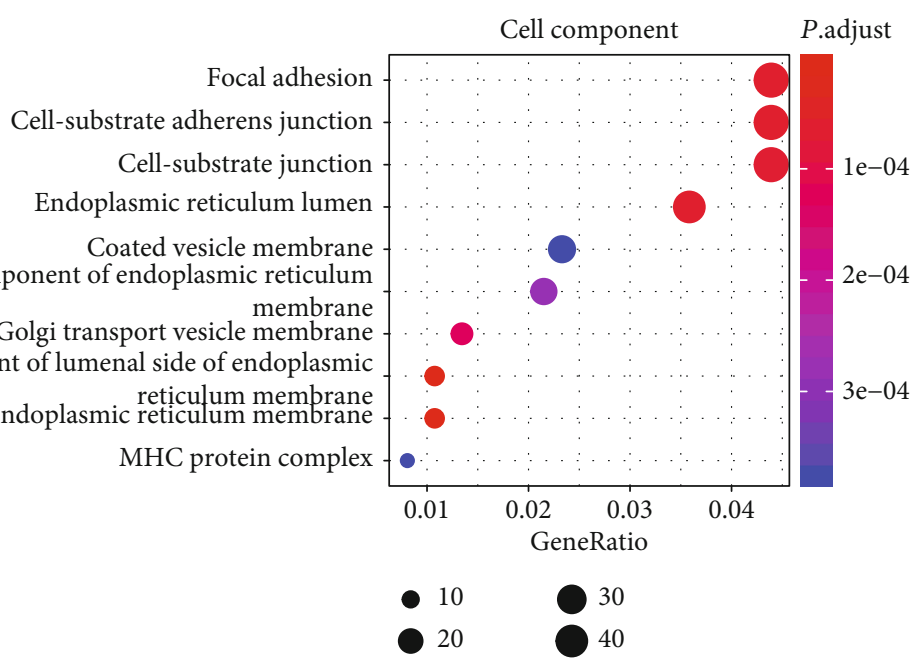

(b)

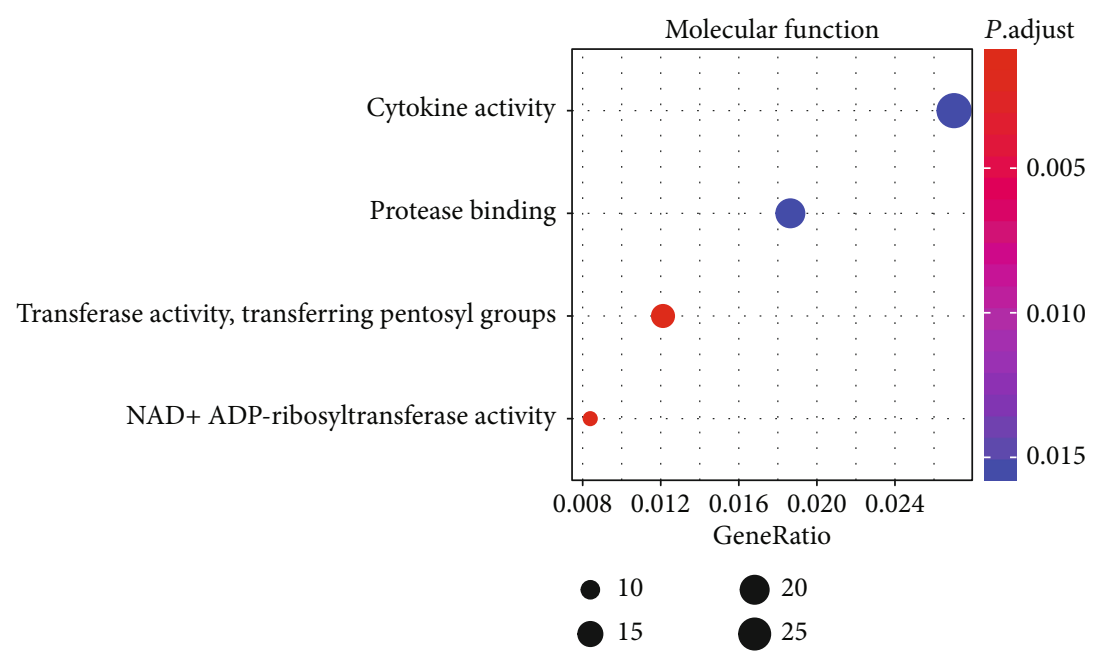

(c)

FIGURE 6: Dot plots for GO enrichment analysis of DEGs between the OV-BRCA1 and NC groups. (a) Dot plot of the top 10 GO terms in the biological process group. (b) Dot plot of the top $10 \mathrm{GO}$ terms in the cell component group. (c) Dot plot of the top 4 GO terms in the molecular function group. The gradual color of the dots indicates the adjusted $P$ value, and the size of the dots indicates the number of genes in the dot plots. 


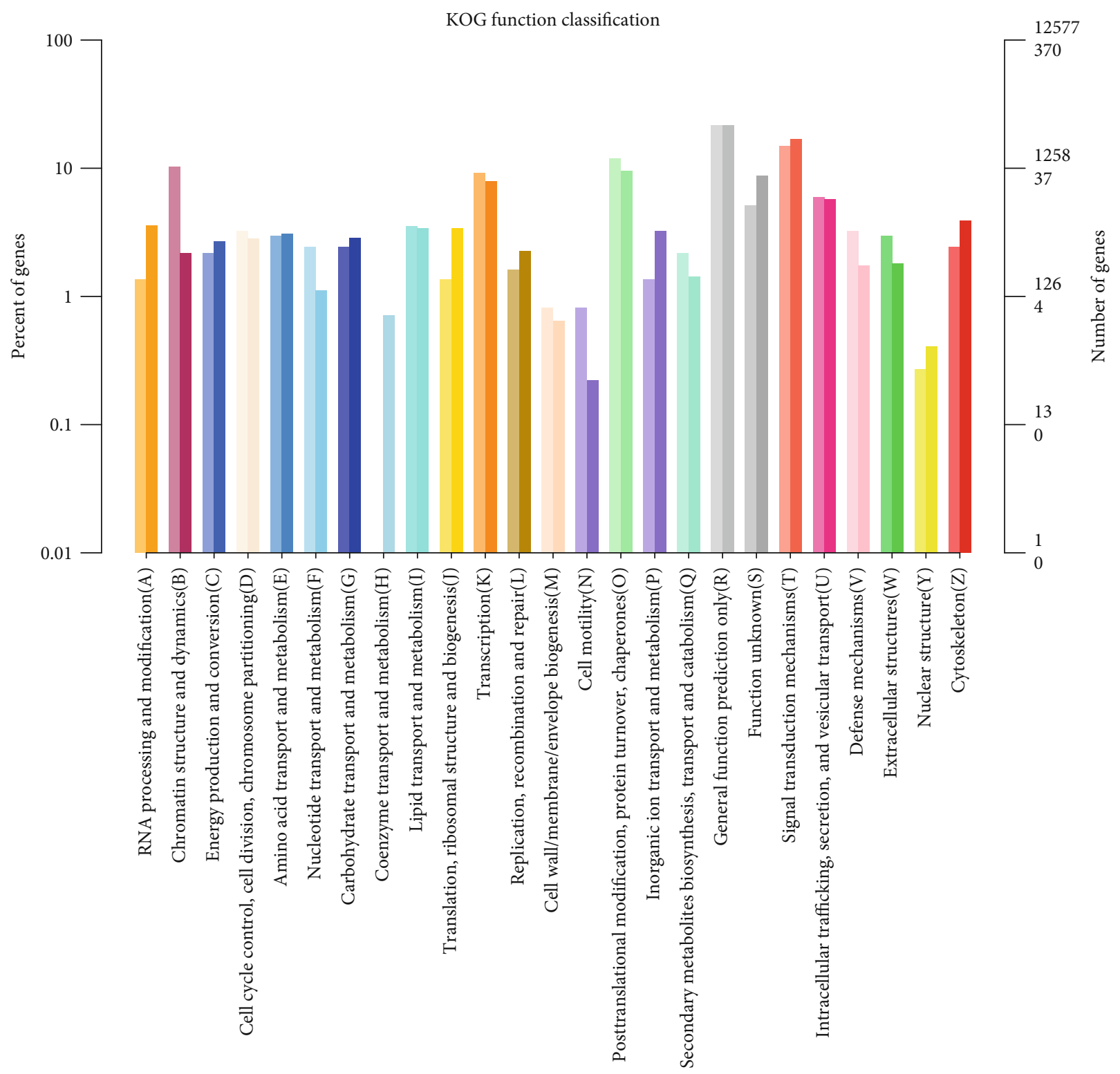

FIgURE 7: Functional classification of euKaryotic Ortholog Groups (KOG) database on DEGs. The $x$-axis shows the contents of KOG, the left $y$-axis shows the percent of genes, and the right $y$-axis shows the number of genes in the classification.

BRCA1 (BRCA1 DNA repair associated) is a tumor suppressor gene and related to hereditary breast and ovarian cancer. BRCA1 plays a pivotal role in homologous recombination-based DNA repair and functions in both cell cycle checkpoint control and maintenance of genomic stability [30]. Genomic instability caused by DNA repair defect after BRCA1 deletion may lead to tumorigenesis [31, 32]; however, it was also because of DNA repair defect in BRCA1-deficient cells, DNA damage caused by platinumbased chemotherapy or radiation may be substantially enhanced [33-35]. Gao et al. found that advanced or metastatic esophageal squamous cell carcinoma patients with low BRCA1 mRNA expression had increased response rate to cisplatin-based chemotherapy or chemoradiotherapy [36]. For breast cancer and ovarian cancer patients, BRCA1 mutations also increased chemosensitivity and/or radiosensitivity $[37,38]$. Our study showed that the expression level of BRCA1 was higher in CSCC resistant to CCRT than that in CSCC sensitive to CCRT by bioinformatics analysis and qPCR verification. ROC curves showed that BRCA1 showed a high diagnostic value in CSCC patients with CR to CCRT, which demonstrated that BRCA1 might be correlated with the sensitivity of CC cells to CCRT.

Through the analysis of our sequencing results, we found that STAT1, STAT2, and CCND1 were upregulated in $\mathrm{SiHa}$ cells with BRCA1 overexpression, and these findings might reveal part of the mechanisms by which BRCA1 affects sensitivity to chemoradiotherapy in CSCC. Previous studies found that BRCA1 is required for the upregulation of STAT1 and STAT2, and they play a synergistic role in the differential 


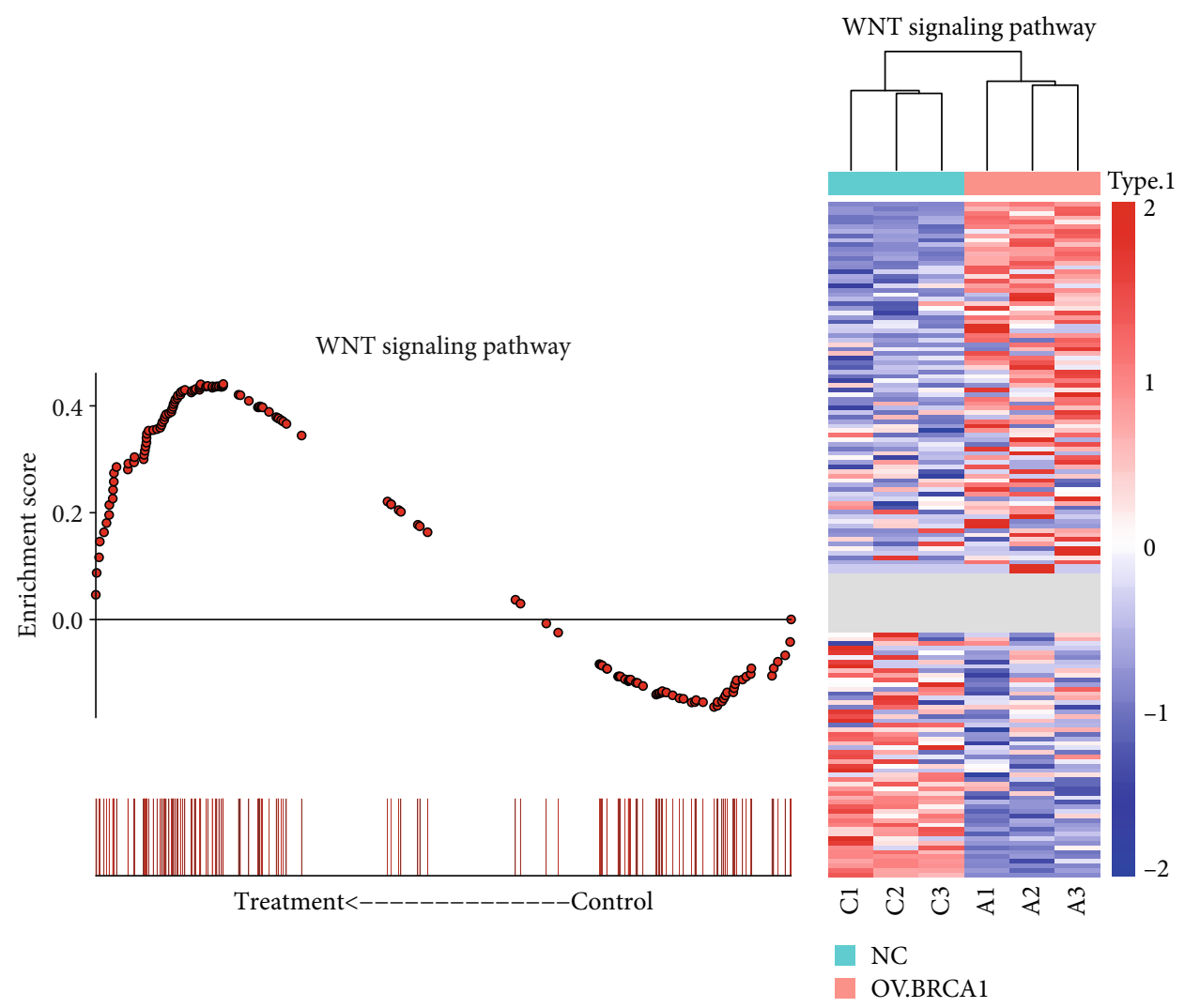

(a)

(b)

WNT signaling pathway

\begin{tabular}{|l|l|l|l|l|l|}
\hline FOSL1 & 0.96 & 0.95 & 0.92 & -0.92 & -0.83 \\
\hline & FZD9 & 0.98 & 0.92 & -0.92 & -0.9 \\
\hline & & & & & \\
\hline & & & & & \\
\hline
\end{tabular}

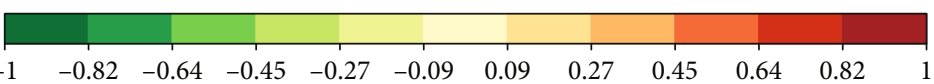

(c)

Figure 8: Continued. 


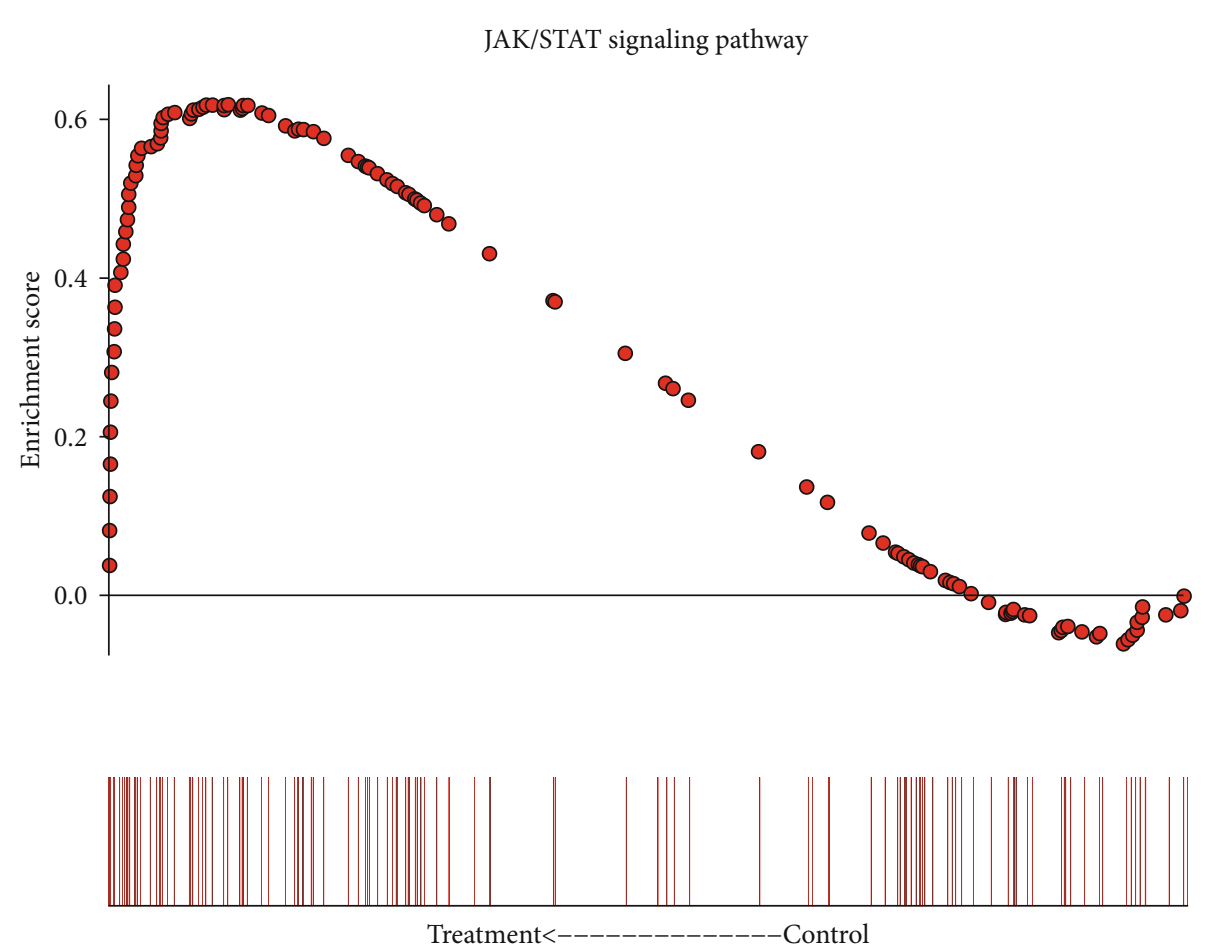

JAK-STAT signaling pathway

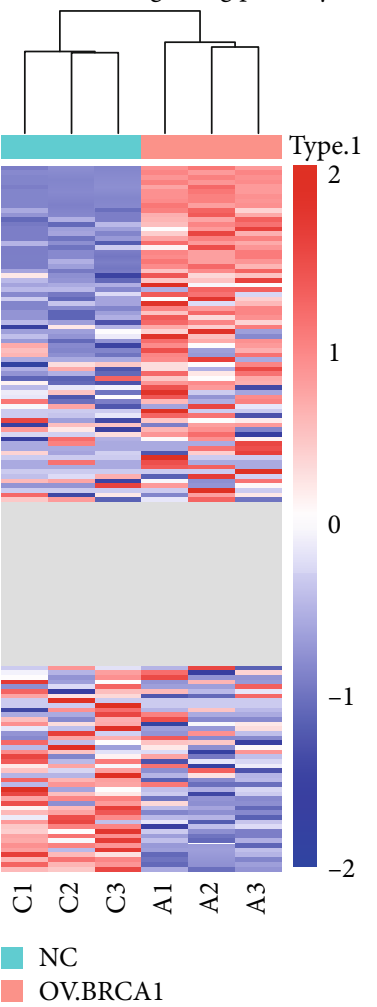

(d)

(e)

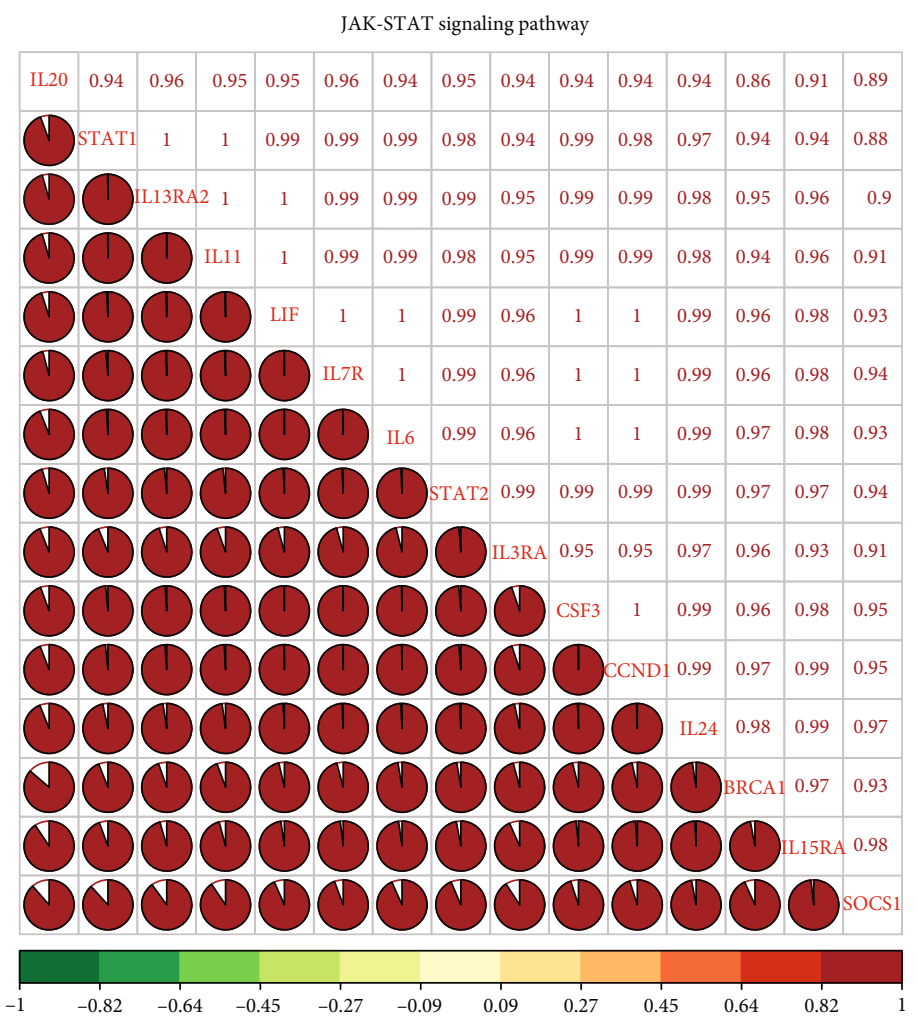

(f)

FIGURE 8: Comprehensive analysis of the WNT and JAK/STAT signaling pathway. (a, d) Enrichment plot of the WNT and JAK/STAT signaling pathway from GSEA. (b, e) The heat map of genes in the WNT and JAK/STAT signaling pathway based on high-throughput sequencing data. (c, f) The relationship between BRCA1 and the key DEGs in the WNT and JAK/STAT signaling pathway. 


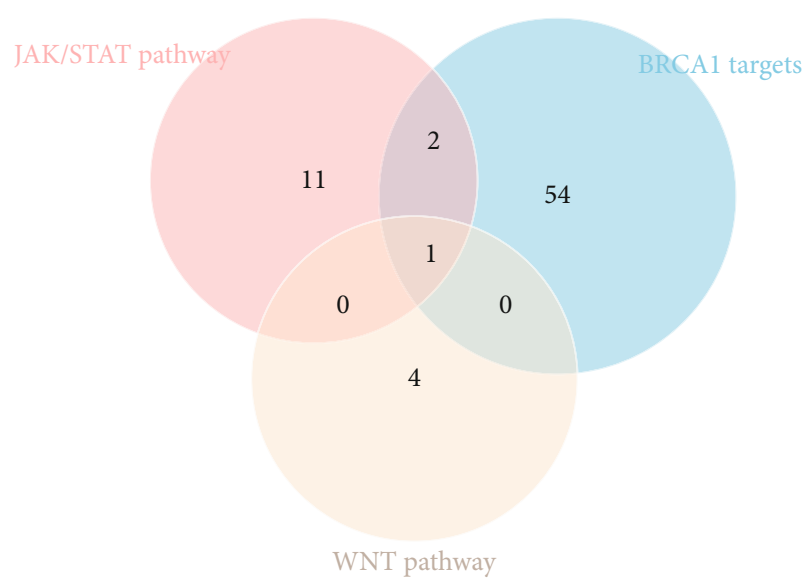

(a)

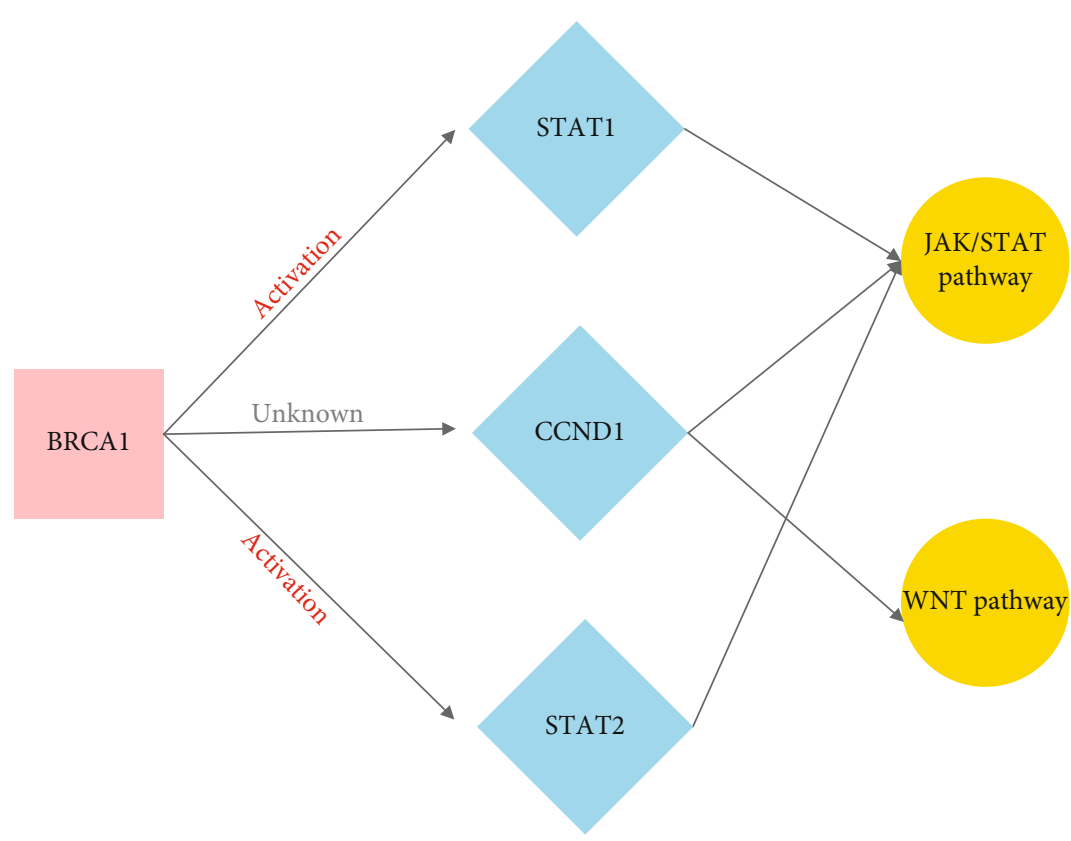

(b)
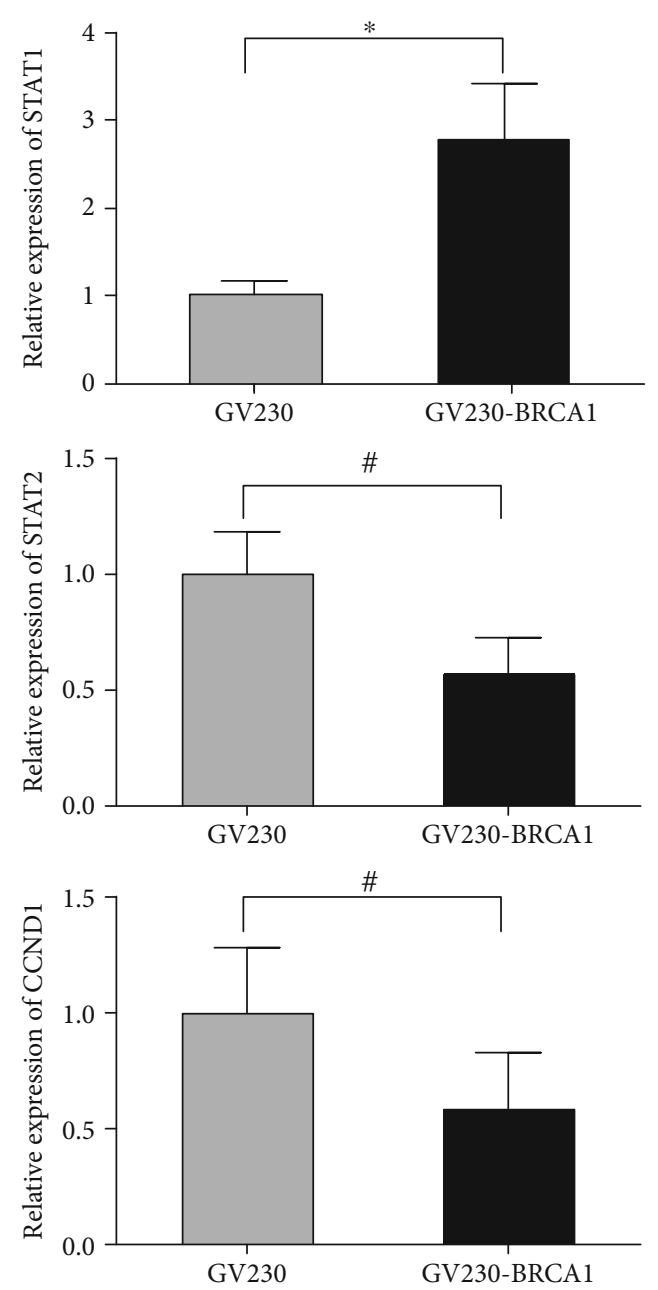

(c)

FIGURE 9: Screening of BRCA1-related CCRT resistance mechanisms in CSCC. (a) The Venn analysis of differentially expressed target genes of BRCA1. (b) The potential regulatory network of BRCA1 in the CCRT resistance of CSCC. (c) The expression of STAT1, STAT2, and CCND1 by qRT-PCR in the SiHa cell with GV230-BRCA1 or GV230 transfection.

regulation of IFN-gamma target genes $[39,40]$. In breast cancer patients who are BRCA1 mutation carriers, CCND1 expression was significantly reduced [41]. These findings are consistent with our results, demonstrating that BRCA1 expression is positively correlated with STAT1, STAT2, and CCND1. STAT1 and STAT2 are important components of the JAK/STAT pathway, and continued activation of the JAK/STAT signal is associated with tumor progression [42, 43]. CCND1 is often overexpressed in tumors, and its expression is positively correlated with activation of the JAK/STAT pathway [44]. In addition, studies showed the JAK/STAT pathway played a crucial role in cisplatin resistance of CC $[45,46]$. Furthermore, our qPCR validation revealed that STAT1 was upregulated in SiHa cells overexpressing BRCA1 in our study. We speculated that BRCA1 may increase the sen- sitivity of CSCC patients to cisplatin-based CCRT by upregulating STAT1 to activate the JAK/STAT pathway.

\section{Conclusion}

Our work analyzed transcriptome-related changes in CCRTresistant CSCC patients and $\mathrm{SiHa}$ cells based on GEO dataset analysis and transcriptome sequencing. We found that BRCA1 may enhance the sensitivity of CSCC patients to cisplatin-based CCRT by upregulating STAT1 to activate the JAK/STAT pathway. These findings may provide new therapeutic strategies to overcome intrinsic or acquired CCRT resistance in CSCC. Additional studies are needed to further elucidate the specific mechanism of BRCA1 in CCRT resistance of CSCC. 


\section{Data Availability}

The following information was supplied regarding data availability. The transcriptome sequencing data has been uploaded to the GEO database, and the accession number is GSE141558. Expression profiles of GSE56363 in the manuscript were downloaded from NCBI-GEO (https://www .ncbi.nlm.nih.gov/gds).

\section{Conflicts of Interest}

The authors declare that they have no conflicts of interest.

\section{Authors' Contributions}

All authors were involved in the study and approved the final version of the manuscript.

\section{Acknowledgments}

This study was supported by grants from the National Natural Science Foundation of China (No. 81772772) and the Medical and Health Talents Project of Jilin Province (2019SCZT003).

\section{Supplementary Materials}

Table S1: differentially expressed genes in the BRCA1 overexpression group compared with control group. Table S2: summary of sequencing data. (Supplementary Materials)

\section{References}

[1] F. Bray, J. Ferlay, I. Soerjomataram, R. L. Siegel, L. A. Torre, and A. Jemal, "Global cancer statistics 2018: GLOBOCAN estimates of incidence and mortality worldwide for 36 cancers in 185 countries," CA: a Cancer Journal for Clinicians, vol. 68, no. 6, pp. 394-424, 2018.

[2] W. Small Jr., M. A. Bacon, A. Bajaj et al., "Cervical cancer: a global health crisis," Cancer, vol. 123, no. 13, pp. 2404-2412, 2017.

[3] E. L. van Dijk, H. Auger, Y. Jaszczyszyn, and C. Thermes, "Ten years of next-generation sequencing technology," Trends in Genetics, vol. 30, no. 9, pp. 418-426, 2014.

[4] E. L. van Dijk, Y. Jaszczyszyn, D. Naquin, and C. Thermes, "The third revolution in sequencing technology," Trends in Genetics, vol. 34, no. 9, pp. 666-681, 2018.

[5] P. A. Bissey, J. H. Law, J. P. Bruce et al., "Dysregulation of the MiR-449b target TGFBI alters the TGF $\beta$ pathway to induce cisplatin resistance in nasopharyngeal carcinoma," Oncogene, vol. 7, no. 5, p. 40, 2018.

[6] D. Jiang, X. Wang, Y. Wang et al., "Mutation in BRAF and SMAD4 associated with resistance to neoadjuvant chemoradiation therapy in locally advanced rectal cancer," Virchows Archiv, vol. 475, no. 1, pp. 39-47, 2019.

[7] Y. Luo, L. Tong, H. Meng et al., "MiR-335 regulates the chemo-radioresistance of small cell lung cancer cells by targeting PARP-1," Gene, vol. 600, pp. 9-15, 2017.

[8] P. F. Cosper, C. McNair, I. Gonzalez et al., "Decreased local immune response and retained HPV gene expression during chemoradiotherapy are associated with treatment resistance and death from cervical cancer," International Journal of Cancer, vol. 146, no. 7, pp. 2047-2058, 2019.

[9] K. Zempolich, C. Fuhrman, B. Milash et al., "Changes in gene expression induced by chemoradiation in advanced cervical carcinoma: a microarray study of RTOG C-0128," Gynecologic Oncology, vol. 109, no. 2, pp. 275-279, 2008.

[10] R. D. Kennedy, J. E. Quinn, P. G. Johnston, and D. P. Harkin, "BRCA1: mechanisms of inactivation and implications for management of patients," The Lancet, vol. 360, no. 9338, pp. 1007-1014, 2002.

[11] R. D. Kennedy, J. E. Quinn, P. B. Mullan, P. G. Johnston, and D. P. Harkin, "The role of BRCA1 in the cellular response to chemotherapy," Journal of the National Cancer Institute, vol. 96, no. 22, pp. 1659-1668, 2004.

[12] L. Bonanno, C. Costa, M. Majem et al., "Combinatory effect of BRCA1 and HERC2 expression on outcome in advanced non-small-cell lung cancer," BMC Cancer, vol. 16, no. 1, 2016.

[13] J. L. Lesnock, K. M. Darcy, C. Tian et al., "BRCA1 expression and improved survival in ovarian cancer patients treated with intraperitoneal cisplatin and paclitaxel: a Gynecologic Oncology Group Study," British Journal of Cancer, vol. 108, no. 6, pp. 1231-1237, 2013.

[14] C. Sun, N. Li, Z. Yang et al., "miR-9 regulation of BRCA1 and ovarian cancer sensitivity to cisplatin and PARP inhibition," Journal of the National Cancer Institute, vol. 105, no. 22, pp. 1750-1758, 2013.

[15] J. M. Hair, G. I. Terzoudi, V. I. Hatzi et al., "BRCA1 role in the mitigation of radiotoxicity and chromosomal instability through repair of clustered DNA lesions," Chemico-Biological Interactions, vol. 188, no. 2, pp. 350-358, 2010.

[16] X. He and S. Fan, "hsa-miR-212 modulates the radiosensitivity of glioma cells by targeting BRCA1," Oncology Reports, vol. 39, no. 3, pp. 977-984, 2017.

[17] K. Söderlund, L. Skoog, T. Fornander, and M. S. Askmalm, "The BRCA1/BRCA2/Rad 51 complex is a prognostic and predictive factor in early breast cancer," Radiotherapy and Oncology, vol. 84, no. 3, pp. 242-251, 2007.

[18] O. Balacescu, L. Balacescu, O. Tudoran et al., "Gene expression profiling reveals activation of the FA/BRCA pathway in advanced squamous cervical cancer with intrinsic resistance and therapy failure," BMC Cancer, vol. 14, no. 1, 2014.

[19] M. E. Ritchie, B. Phipson, D. Wu et al., "limma powers differential expression analyses for RNA-sequencing and microarray studies," Nucleic Acids Research, vol. 43, no. 7, pp. e47-e47, 2015.

[20] G. Yu, L. G. Wang, Y. Han, and Q. Y. He, "clusterProfiler: an R package for comparing biological themes among gene clusters," OMICS, vol. 16, no. 5, pp. 284-287, 2012.

[21] D. Szklarczyk, A. Franceschini, S. Wyder et al., "STRING v10: protein-protein interaction networks, integrated over the tree of life," Nucleic Acids Research, vol. 43, no. D1, pp. D447D452, 2015.

[22] P. Shannon, A. Markiel, O. Ozier et al., "Cytoscape: a software environment for integrated models of biomolecular interaction networks," Genome Research, vol. 13, no. 11, pp. 2498 2504, 2003.

[23] C.-H. Chin, S.-H. Chen, H.-H. Wu, C.-W. Ho, M.-T. Ko, and C.-Y. Lin, "cytoHubba: identifying hub objects and subnetworks from complex interactome," Bmc Systems Biology, vol. 8, Supplement 4, p. S11, 2014. 
[24] X. Robin, N. Turck, A. Hainard et al., "pROC: an open-source package for R and S+ to analyze and compare ROC curves," BMC Bioinformatics, vol. 12, no. 1, p. 77, 2011.

[25] H. Wickham, ggplot 2: Elegant Graphics for Data Analysis, Springer, 2016.

[26] R. Kolde, "Pheatmap: pretty heatmaps," R Package Version, vol. 61, no. 926, p. 915, 2012.

[27] S. Anders and W. Huber, Differential expression of RNA-Seq data at the gene level-the DESeq package, European Molecular Biology Laboratory (EMBL), Heidelberg, Germany, 2012.

[28] A. Subramanian, P. Tamayo, V. K. Mootha et al., "Gene set enrichment analysis: a knowledge-based approach for interpreting genome-wide expression profiles," Proceedings of the National Academy of Sciences, vol. 102, no. 43, pp. 1554515550, 2005.

[29] H. Han, J. W. Cho, S. Lee et al., "TRRUST v2: an expanded reference database of human and mouse transcriptional regulatory interactions," Nucleic Acids Research, vol. 46, no. D1, pp. D380-d386, 2018.

[30] B. Wang, S. Matsuoka, B. A. Ballif et al., "Abraxas and RAP80 form a BRCA1 protein complex required for the DNA damage response," Science, vol. 316, no. 5828, pp. 1194-1198, 2007.

[31] M. L. Li and R. A. Greenberg, "Links between genome integrity and BRCA1 tumor suppression," Trends in Biochemical Sciences, vol. 37, no. 10, pp. 418-424, 2012.

[32] A. R. Venkitaraman, "Cancer suppression by the chromosome custodians, BRCA1 and BRCA2," Science, vol. 343, no. 6178, pp. 1470-1475, 2014.

[33] R. W. Anantha, S. Simhadri, T. K. Foo et al., "Functional and mutational landscapes of BRCA1 for homology-directed repair and therapy resistance," eLife, vol. 6, 2017.

[34] C. J. Lord and A. Ashworth, "BRCAness revisited," Nature Reviews. Cancer, vol. 16, no. 2, pp. 110-120, 2016.

[35] A. Ray Chaudhuri, E. Callen, X. Ding et al., "Replication fork stability confers chemoresistance in BRCA-deficient cells," Nature, vol. 535, no. 7612, pp. 382-387, 2016.

[36] Y. Gao, J. Zhu, X. Zhang et al., "BRCA1 mRNA expression as a predictive and prognostic marker in advanced esophageal squamous cell carcinoma treated with cisplatin- or docetaxelbased chemotherapy/chemoradiotherapy," PLoS One, vol. 8, no. 1, article e52589, 2013.

[37] A. Fourquet, D. Stoppa-Lyonnet, Y. M. Kirova, B. Sigal-Zafrani, and B. Asselain, "Familial breast cancer: clinical response to induction chemotherapy or radiotherapy related to BRCA1/2 mutations status," American Journal of Clinical Oncology, vol. 32, no. 2, pp. 127-131, 2009.

[38] J. Weberpals, K. Garbuio, A. O'Brien et al., “The DNA repair proteins BRCA1 and ERCC1 as predictive markers in sporadic ovarian cancer," International Journal of Cancer, vol. 124, no. 4, pp. 806-815, 2009.

[39] N. E. Buckley, A. M. Hosey, J. J. Gorski et al., "BRCA1 regulates IFN- $\gamma$ Signaling through a mechanism involving the type I IFNs," Molecular Cancer Research, vol. 5, no. 3, pp. 261-270, 2007.

[40] T. Ouchi, S. W. Lee, M. Ouchi, S. A. Aaronson, and C. M. Horvath, "Collaboration of signal transducer and activator of transcription 1 (STAT1) and BRCA1 in differential regulation of IFN-gamma target genes," Proceedings of the National Academy of Sciences of the United States of America, vol. 97, no. 10 , pp. 5208-5213, 2000.
[41] J. E. Armes, L. Trute, D. White et al., "Distinct molecular pathogeneses of early-onset breast cancers in BRCA1 and BRCA2 mutation carriers: a population-based study," Cancer Research, vol. 59, no. 8, pp. 2011-2017, 1999.

[42] B. Groner and V. von Manstein, "Jak Stat signaling and cancer: opportunities, benefits and side effects of targeted inhibition," Molecular and Cellular Endocrinology, vol. 451, pp. 1-14, 2017.

[43] J. Pencik, H. T. T. Pham, J. Schmoellerl et al., "JAK-STAT signaling in cancer: from cytokines to non-coding genome," Cytokine, vol. 87, pp. 26-36, 2016.

[44] Z. Li, J. Shen, W. K. K. Wu et al., "Leptin induces cyclin D1 expression and proliferation of human nucleus pulposus cells via JAK/STAT, PI3K/Akt and MEK/ERK pathways," PLoS One, vol. 7, no. 12, article e53176, 2012.

[45] L. L. Huang and W. Rao, "SiRNA interfering STAT3 enhances DDP sensitivity in cervical cancer cells," European Review for Medical and Pharmacological Sciences, vol. 22, no. 13, pp. 4098-4106, 2018.

[46] R. Stroeder, B. W. Rückheim, J. Fischbach et al., "Oncostatin M treatment increases the responsiveness toward cisplatin-based chemoradiotherapy in cervical cancer cells in a STAT3dependent manner," Oncology Letters, vol. 16, no. 3, pp. 3351-3358, 2018. 